\title{
Effect of leptin treatment in a mouse model of TDP-43 proteinopathy
}

Agueda Ferrer-Donato ${ }^{\mathrm{a}, \uparrow}$, Ana Contreras ${ }^{\mathrm{b}, \dagger}$, Paloma Fernandez ${ }^{\mathrm{c}}$, Carmen M. Fernandez$\operatorname{Martos}^{\mathrm{a}, \mathrm{d}, *}$.

${ }^{a}$ Research Unit of the National Hospital of Paraplegics (UDI-HNP), Toledo, Spain.

aferrerd@externas.sescam.jccm.es (A.F.-D.); Carmen.fernandezmartos@utas.edu.au (CM.F.M.).

${ }^{b}$ Health Research Centre (CEINSA), University of Almeria (UAL), Almeria, Spain. Acm699@ual.es (A.C.)

${ }^{c}$ Institute of Applied Molecular Medicine (IMMA), Faculty of Medicine, Universidad San Pablo CEU, Madrid, Spain.paloma.fernandezmartinez@ceu.es (P.F.).

${ }^{d}$ Wicking Dementia Research and Education Centre, College of Health and Medicine, University of Tasmania, Hobart, Tasmania, Australia.Carmen.fernandezmartos@ utas.edu.au (CM.F.-M.).

†These authors are both last authors.

\section{*Corresponding author:}

Dr. Carmen M. Fernandez-Martos.

Primary Email: cmfernandezm@sescam.jccm.es.

Secondary Email: Carmen.fernandezmartos@utas.edu.au.

Finca La Peraleda s/n, Toledo 45007, Spain.

Phone: +34 925247789 .

Fax: +34925 247745 .

Abstract: Amyotrophic Lateral Sclerosis (ALS) is an irreversible neurodegenerative disease with no known cure. Recent studies suggest a strong metabolic component in ALS pathogenesis and have shown an inverse relationship between leptin levels and ALS progression, although the effects of leptin as a treatment have not yet been studied. Therefore, we aim to examine whether the acute treatment with leptin has beneficial effects on brain pathology and cognitive function in the transgenic TDP43 ${ }^{\mathrm{A} 315 \mathrm{~T}}$ line of ALS. Mice were treated intranasally (IN) with $0.03 \mathrm{mg} / \mathrm{kg}$ of leptin or vehicle (VH) daily for 7 days. Data showed a progressive decline in body weight and motor coordination in TDP43 ${ }^{\mathrm{A} 315 \mathrm{~T}}$ mice. Moreover, Lep-treated TDP43 ${ }^{\mathrm{A} 315 \mathrm{~T}}$ mice showed an earlier disease 
onset, along with an improvement in motor performance. Altered levels of some of the adipokines and metabolic proteins studied were found in TDP43 ${ }^{\mathrm{A} 315 \mathrm{~T}}$ mice, which were differently expressed among Lep-treated and VH-treated animals. Furthermore, some correlations were found among the serum levels of this proteins in WT and TDP43 ${ }^{\mathrm{A} 315 \mathrm{~T}}$ mice. As far as we know, this is the first pilot study to provide evidence of the therapeutic effect of leptin treatment in a mice model of ALS, although further studies are needed to expound on the underlying mechanisms.

Keywords: Leptin; Metabolism; Amyotrophic Lateral Sclerosis (ALS); TAR DNA binding protein (TDP-43). 


\section{INTRODUCTION}

Amyotrophic Lateral Sclerosis (ALS) is an irreversible neurodegenerative disease characterized by the selective and progressive loss of motor neurons of both the brain and spinal cord [1]. ALS represents a significant socio-economic problem worldwide with prevalence rates of 5 per 100,000 in the USA and 2-3 per 100,000 in Europe [2]. The loss of motor neurons leads to paralysis and, ultimately, death within 3-5 years of symptom onset. There is no known cure for ALS and, currently, there are only two diseasemodifying drugs approved by the USA Food and Drug Administration, riluzole and edaravone, both with modest benefits on survival $[3,4]$.

Recent studies suggest there is a strong metabolic component in ALS pathogenesis, being that the majority of patients show hypermetabolism, rapid weight loss, and low body mass index [5-7]. Moreover, even though obesity represents the second preventable mortality cause worldwide [8], there is growing evidence that being overweight or obese provides a survival advantage in ALS patients [9]. In contrast, the majority of ALS patients (16$55 \%$ ) suffer from malnutrition, which has been identified as a negative prognostic factor $[10,11]$, and is associated with low levels of leptin [12].

Leptin is a hormone secreted mainly by adipocytes, and its traditional role is to regulate food intake and energy balance in the brain $[13,14]$. Nevertheless, the presence of its receptor, $\mathrm{Ob}-\mathrm{Rb}$, in extra-hypothalamic brain areas, suggests the implication of this adipokine in other processes [15]. Thus, leptin plays an important role in several neurological disorders, showing a neuroprotective role in different pathologies, such as Alzheimer's disease [16, 17], Parkinson disease [18], ischemia [19] or spinal cord injuries [20]. Furthermore, it improves memory processes and facilitates synaptic plasticity [21, 22], which indicates the potential role of leptin in preventing cognitive decline. Indeed, leptin had been shown to have an anti-amyloid effect as well as to reduce spatial memory 
deficits in the APP/PS1 transgenic mice model of Alzheimer's disease [23]. Regarding ALS, some epidemiological studies have shown an inverse relationship between leptin levels and ALS risk and progression [24, 25]. Thus, increasing leptin concentrations could lead to slower disease onset and progression. In this sense, some preclinical studies showed that feeding ALS mice with a high-fat diet improves survival and delays neurodegeneration [26] and a hypercaloric diet had shown efficacy in prolonging lifespan in a pilot clinical trial in gastrostomised ALS patients [27], which could be related to leptin levels as weight gain correlates with higher levels of this adipokine [28].

Despite all the data linking leptin with ALS, there are no previous studies that look at the possible therapeutic effect of this hormone. In this context, we aim to conduct a pilot study to examine whether acute treatment with leptin has beneficial effects on brain pathology and motor function in a mice model of TDP-43 proteinopathy, the transgenic TDP43 ${ }^{\mathrm{A} 315 \mathrm{~T}}$ line of ALS. Our results show that leptin therapy significantly improved motor performance and support the neuroprotective role of leptin in ALS.

\section{RESULTS}

\subsection{Effect of leptin on disease progression}

Body weight loss and motor performance were monitored in both TDP-43 ${ }^{\mathrm{A} 315 \mathrm{~T}}$ and WT mice exposed to either leptin or $\mathrm{VH}$ to evaluate the effect of treatment on disease progression.

A two-way ANOVA with repeated measures revealed a significant genotype interaction (Figure 1A; $\left.F_{(18,83)}=7.701, p<0.0001\right)$, indicating a sustained decline in body weight in TDP-43 ${ }^{\mathrm{A} 315 \mathrm{~T}}$ mice compared to WT in responses to the treatment over time. Although no 
significant difference was found between Lep-treated and VH-treated TDP-43 ${ }^{\mathrm{A} 315 \mathrm{~T}}$ mice, weight loss decline seemed less prominent after leptin treatment until week 11.

Disease onset was calculated using body weight measurements with a criterion of $10 \%$ body weight loss. Results showed that TDP- $43^{\mathrm{A} 315 \mathrm{~T}}$ mice treated with leptin had an earlier onset than TDP-43 ${ }^{\mathrm{A} 315 \mathrm{~T}}$ mice treated with VH (Figure $\left.1 \mathrm{~B} ; \mathrm{t}_{5}=5.576 \mathrm{p}<0.01\right)$. An average, disease onset was $86 \pm 2.5$ days of age for VH-treated TDP-43 ${ }^{\mathrm{A} 315 \mathrm{~T}}$ mice whereas Leptreated TDP- $43^{\mathrm{A} 315 \mathrm{~T}}$ mice showed the phenotype at $68 \pm 1.7$ days of age.

Additionally, when analysing disease duration- calculated as the time between disease onset and disease end-stage- data showed a longer disease duration in TDP-43 ${ }^{\mathrm{A} 315 \mathrm{~T}}$ mice after leptin treatment compared to $\mathrm{VH}$ treatment (Figure $1 \mathrm{C} ; \mathrm{t}_{4}=4.808 \mathrm{p}<0.01$ ). Nevertheless, life expectancy was similar among the two groups ( 95-100 \pm 2 days).

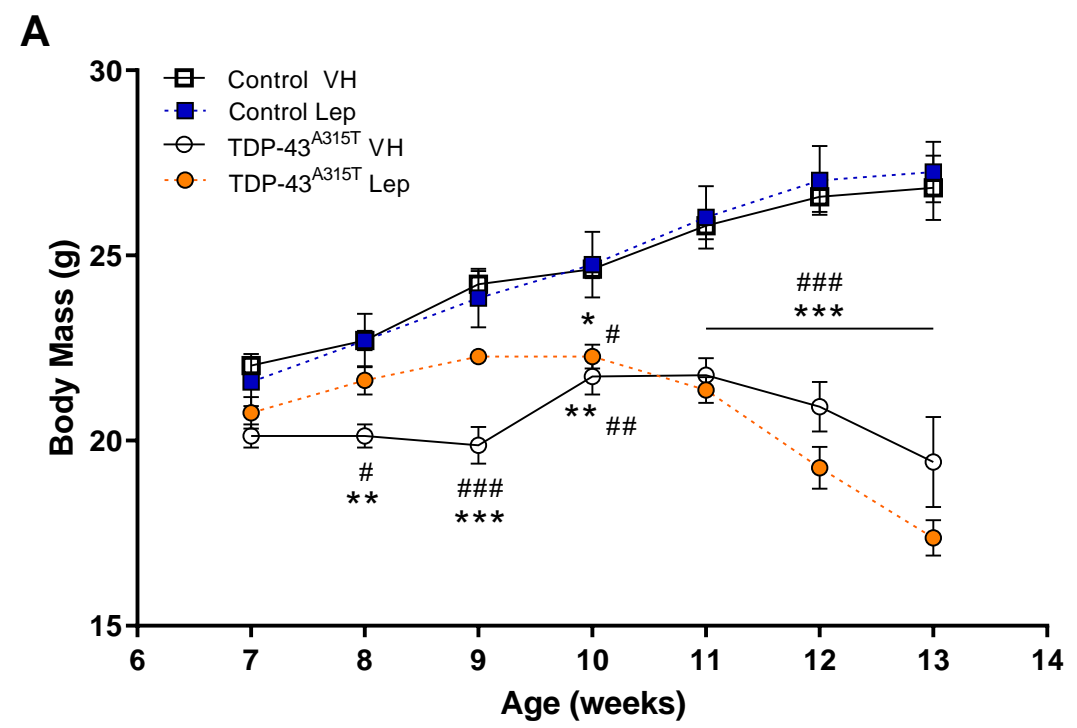

B

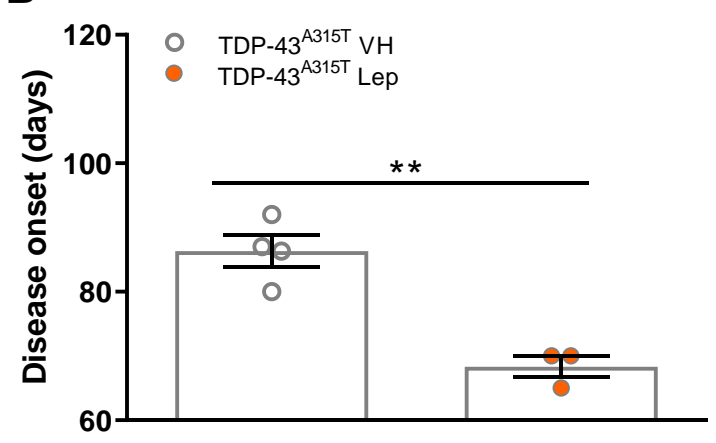

Body weight (g)
C

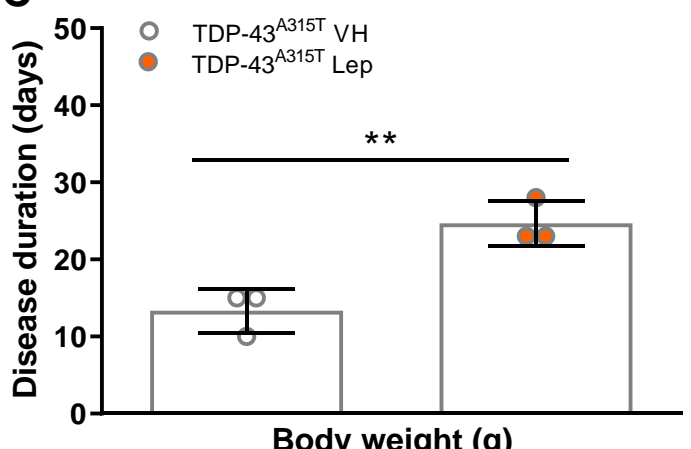


Figure 1. Leptin treatment does not alter body weight loss but changes disease onset and duration in TDP-43 $\mathbf{4 3}^{\mathrm{A315T}}$ mice. Values are expressed as mean \pm SEM for the different groups: TDP-43 ${ }^{\mathrm{A} 315 \mathrm{~T}}$ Lep (orange circles, $\mathrm{n}=3$ ), TDP-43 ${ }^{\mathrm{A} 315 \mathrm{~T}} \mathrm{VH}$ (white circles, $\mathrm{n}=5$ ), Control Lep (blue square, $\mathrm{n}=4$ ), and Control VH (white square, $n=4$ ). (A) Body weight was monitored over time in WT and TDP-43 ${ }^{\mathrm{A} 315 \mathrm{~T}}$ mice exposed to either leptin or VH. No significant differences were observed between Lep-exposed or VH-exposed TDP- $43^{\mathrm{A} 315 \mathrm{~T}}$ mice. $* \mathrm{p}<0.05, * * \mathrm{p}<0.01, * * * \mathrm{p}<0.001$ vs. Control VH; \# $\mathrm{p}<0.05$, \#\# $\mathrm{p}<0.01$, \#\#\# p<0.001 vs. Control Lep. (B) Average disease onset was determined using body weight as a physiological parameter with a $10 \%$ of normal body weight criterion. $* * \mathrm{p}<0.01$ vs. TDP-43 ${ }^{\mathrm{A} 315 \mathrm{~T}}$ VH. (C) Average disease duration was calculated using body weight as well, as the difference between disease onset and end-stage. $* * p<0.01$ vs. TDP$43^{\mathrm{A} 315 \mathrm{~T}} \mathrm{VH}$.

Moreover, data from the rota-rod test showed a progressive decline in motor coordination in TDP-43 ${ }^{\mathrm{A} 315 \mathrm{~T}}$ mice. A two-way ANOVA with repeated measures revealed a significant effect by week (Figure $2 ; F_{(5,85)}=10.36 \mathrm{p}<0.0001$ ), indicating differential change over time in motor performance. Furthermore, there was a significant effect by treatment $\left(\mathrm{F}_{(3,85)}=56.89 \mathrm{p}<0.0001\right)$, indicating that TDP-43 ${ }^{\mathrm{A} 315 \mathrm{~T}}$ mice treated with leptin showed a significant improvement compared to TDP-43 ${ }^{\mathrm{A} 315 \mathrm{~T}}$ mice treated with $\mathrm{VH}$.

Although leptin treatment caused no significant improvement in weight loss in TDP$43^{\mathrm{A} 315 \mathrm{~T}}$ mice, it showed a protective role on motor function and coordination.

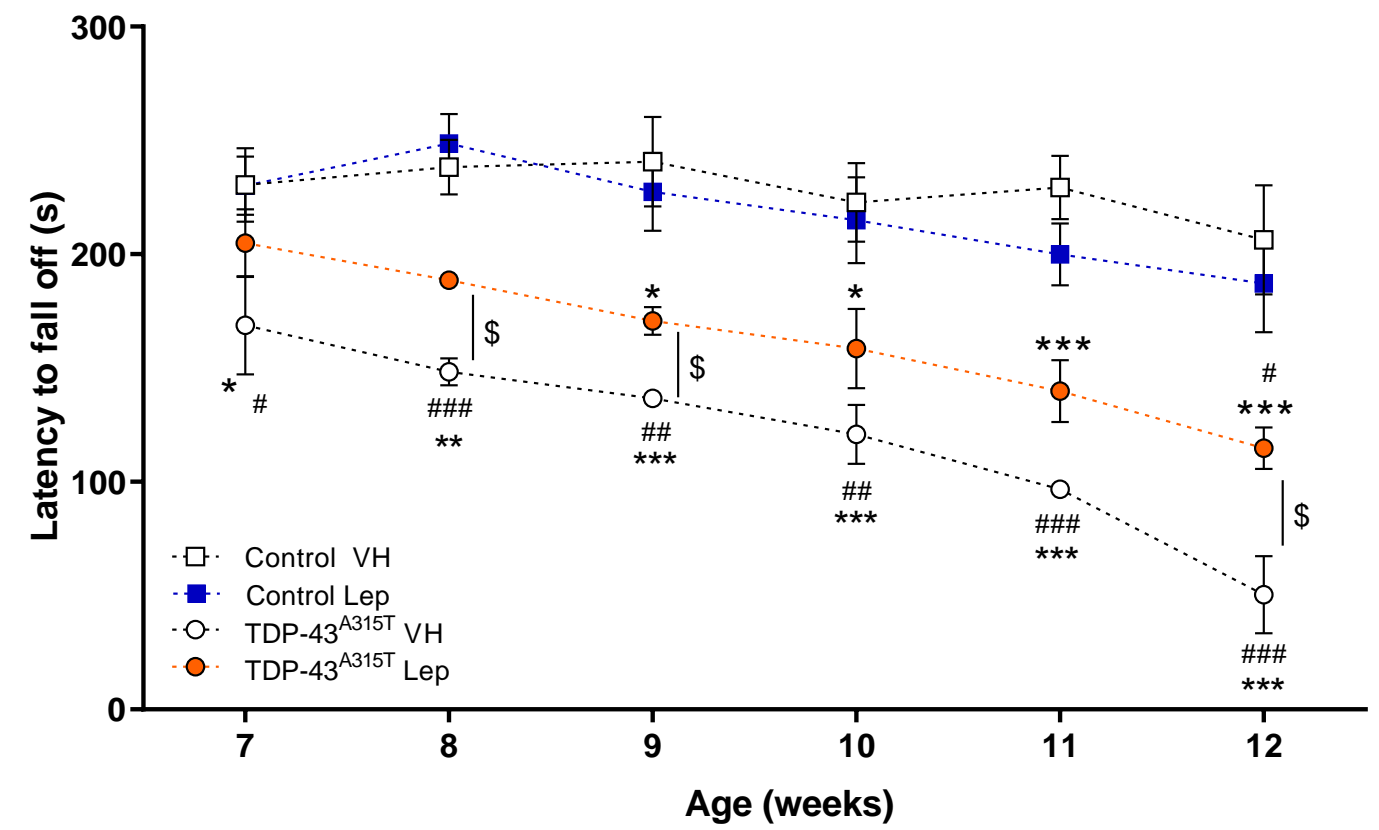


Figure 2. Leptin treatment significantly improves motor performance in TDP$\mathbf{4 3}^{\text {A315T }}$ mice. Values are expressed as mean \pm SEM for the different groups: TDP-43 ${ }^{\text {A315T }}$ Lep (orange circles, $n=3$ ), TDP-43 ${ }^{\mathrm{A} 315 \mathrm{~T}} \mathrm{VH}$ (white circles, $\mathrm{n}=5$ ), Control Lep (blue square, $\mathrm{n}=4$ ) and Control VH (white square, $\mathrm{n}=4$ ). $* \mathrm{p}<0.05$, ** $\mathrm{p}<0.01$, *** $\mathrm{p}<0.001$ vs. Control VH; \# $\mathrm{p}<0.05$, \#\# $\mathrm{p}<0.01$, \#\#\# $\mathrm{p}<0.001$ vs. Control Lep; $\$ \mathrm{p}<0.05$ vs. TDP$43^{\mathrm{A} 315 \mathrm{~T}} \mathrm{VH}$.

\subsection{Effect of leptin on adipokines and metabolic biomarkers}

As metabolic homeostasis is unbalanced in ALS patients [5], levels of adipokines and other metabolic proteins were measured to evaluate the effect of leptin treatment in TDP$43^{\mathrm{A} 315 \mathrm{~T}}$ and WT mice.

Results indicated that levels of adipokines in plasma were altered in TDP-43 ${ }^{\mathrm{A} 315 \mathrm{~T}}$ mice compared to WT mice. A one-way ANOVA showed a statistically significant difference in the level of ghrelin (Figure $3 \mathrm{~A} ; \mathrm{F}_{(3,12)}=4.524$, $\mathrm{p}<0.05$ ), which was higher in Lep-treated TDP-43 ${ }^{\mathrm{A} 315 \mathrm{~T}}$ mice. Moreover, there was a tendency due to the treatment within the TDP$43^{\mathrm{A} 315 \mathrm{~T}}$ groups. Regarding resistin and leptin, a one-way ANOVA showed significant differences (Figure 3B and 3C; $F_{(3,12)}=10.31, p<0.01 ; F_{(3,12)}=4.962, p<0.05$, respectively), specifically a down-regulation of both adipokines in TDP-43 ${ }^{\mathrm{A} 315 \mathrm{~T}}$ mice compared to WT animals treated with leptin. However, no difference was found in any of the adipokines studied due to the treatment in the TDP-43 ${ }^{\mathrm{A} 315 \mathrm{~T}}$ mice. Additionally, a positive correlation was found among the serum levels of ghrelin and leptin compared to resistin in WT mice (Figure 4A; $\mathrm{r}=0.81, \mathrm{p}<0.05 ; \mathrm{r}=0.762, \mathrm{p}<0.05$, respectively), yet no correlation was found in any of the adipokines studied for the TDP-43 ${ }^{\mathrm{A} 315 \mathrm{~T}}$ mice (Figure 4B).

Moreover, for the metabolic proteins studied, altered levels of PAI-1 and GIP were observed. A one-way ANOVA showed differences in PAI-1 levels (Figure 3D; $\mathrm{F}_{(3 \text {, }}$ 11) $=5.177, \mathrm{p}<0.05)$, particularly an increase in Lep-treated TDP-43 ${ }^{\mathrm{A} 315 \mathrm{~T}}$ mice, as well as a tendency when comparing both treatments within the TDP-43 ${ }^{\mathrm{A} 315 \mathrm{~T}}$ group. Similar to 
that, GIP was altered (Figure $3 \mathrm{E} ; \mathrm{F}_{(3,11)}=4.241$, $\mathrm{p}<0.05$ ), showing an up-regulation in the Lep-treated TDP-43 ${ }^{\mathrm{A} 315 \mathrm{~T}}$ group compared to VH-treated WT. Once again, no difference was found when comparing TDP-43 ${ }^{\mathrm{A} 315 \mathrm{~T}}$ mice treated with either leptin or $\mathrm{VH}$, nor in the other biomarkers studied. However, a positive correlation was found among the plasmatic levels of GIP and GLP-1 compared to insulin in WT animals (Figure 4C; $r=0.778, p<0.05$; 
$\mathrm{r}=0.786, \mathrm{p}<0.05$, respectively), as well as between GIP and PAI-1 (Figure 4D; $r=0.881$, $\mathrm{p}<0.01$ ) and GLP-1 and glucagon (Figure 4D; $r=0.762, \mathrm{p}<0.05$ ) in TDP-43 ${ }^{\mathrm{A} 315 \mathrm{~T}}$ mice.

A

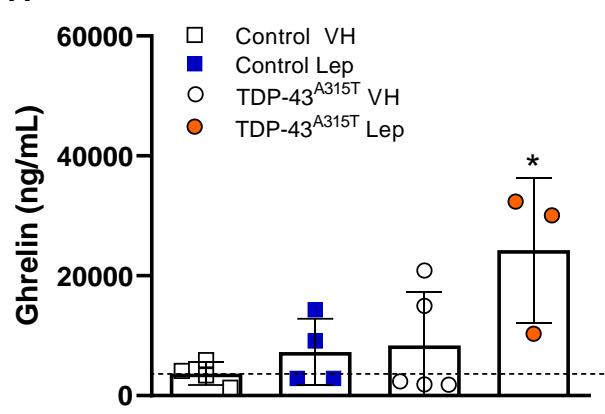

C

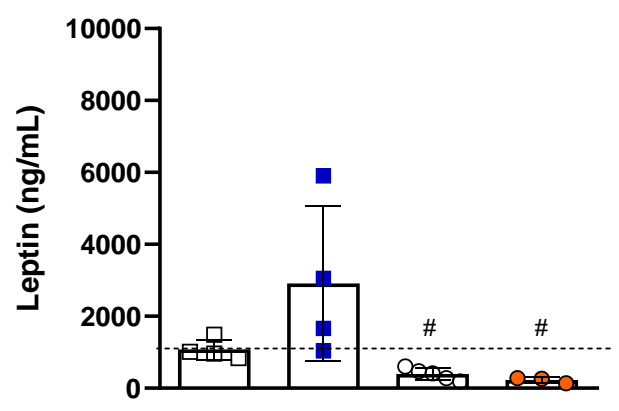

E

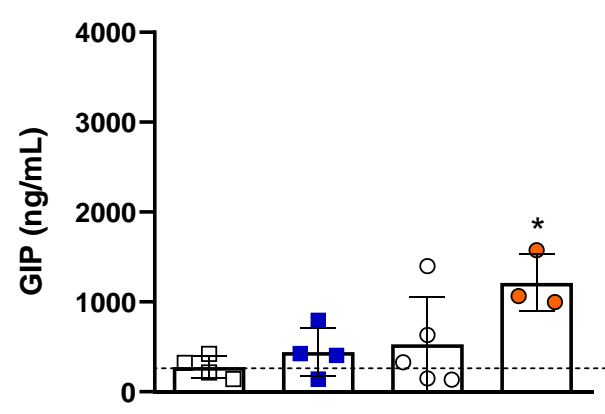

G

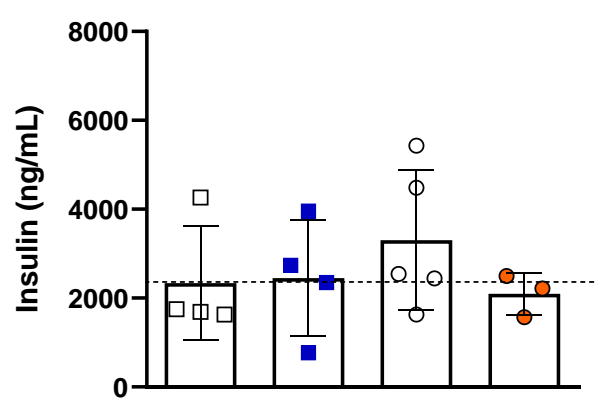

B

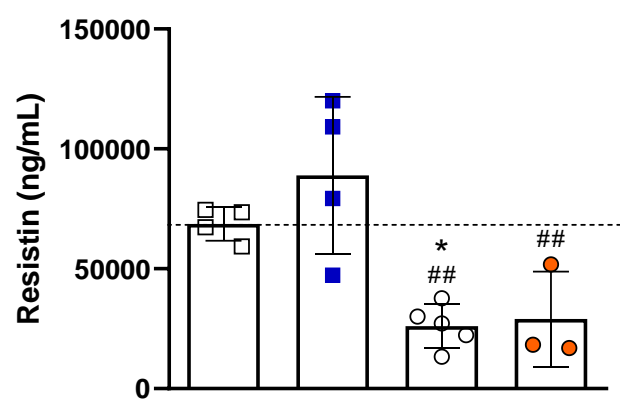

D

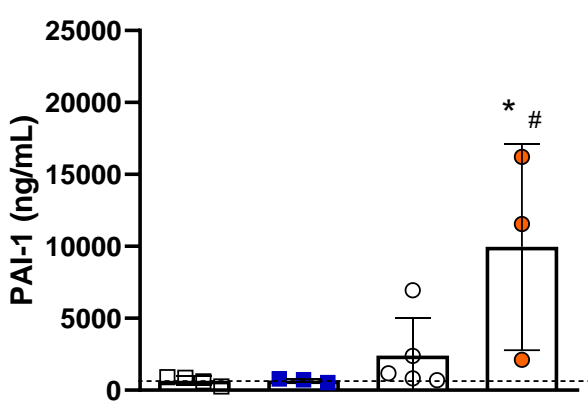

$\mathbf{F}$

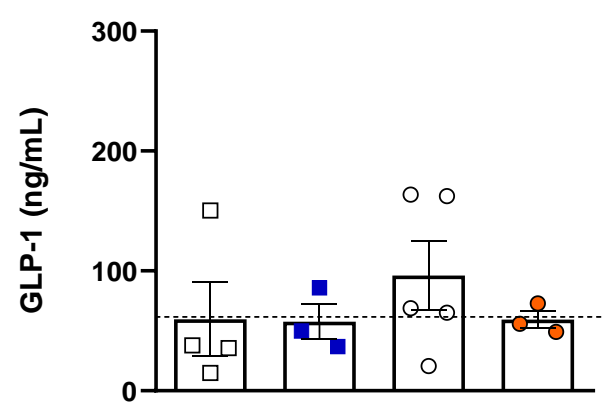

H

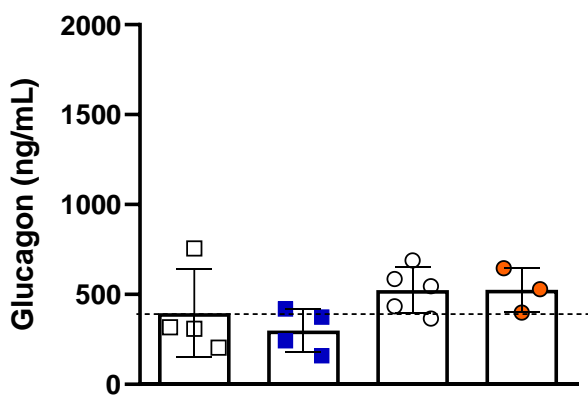

Figure 3. Adipokines and metabolic biomarkers of insulin resistance levels are altered by leptin treatment. Values are expressed as mean \pm SEM for the different groups: TDP-43 ${ }^{\mathrm{A} 315 \mathrm{~T}}$ Lep (orange circles, $\mathrm{n}=3$ ), TDP-43 ${ }^{\mathrm{A} 315 \mathrm{~T}} \mathrm{VH}$ (white circles, $\mathrm{n}=5$ ), Control Lep (blue square, $n=4$ ), and Control VH (white square, $n=4$ ). (A) Ghrelin 
plasmatic concentration. $* \mathrm{p}<0.05$ vs. Control VH. (B) Resistin levels. $* \mathrm{p}<0.05$ vs. Control VH; \#\# p<0.01 vs. Control Lep. (C) Leptin levels. \# p<0.05 vs. Control Lep. (D) PAI-1 levels. * p $<0.05$ vs. Control VH; \# p<0.05 vs. Control Lep. (E) GIP levels. $* \mathrm{p}<0.05$ vs. Control VH. (F-H) GLP-1, insulin, and glucagon plasmatic levels, respectively.
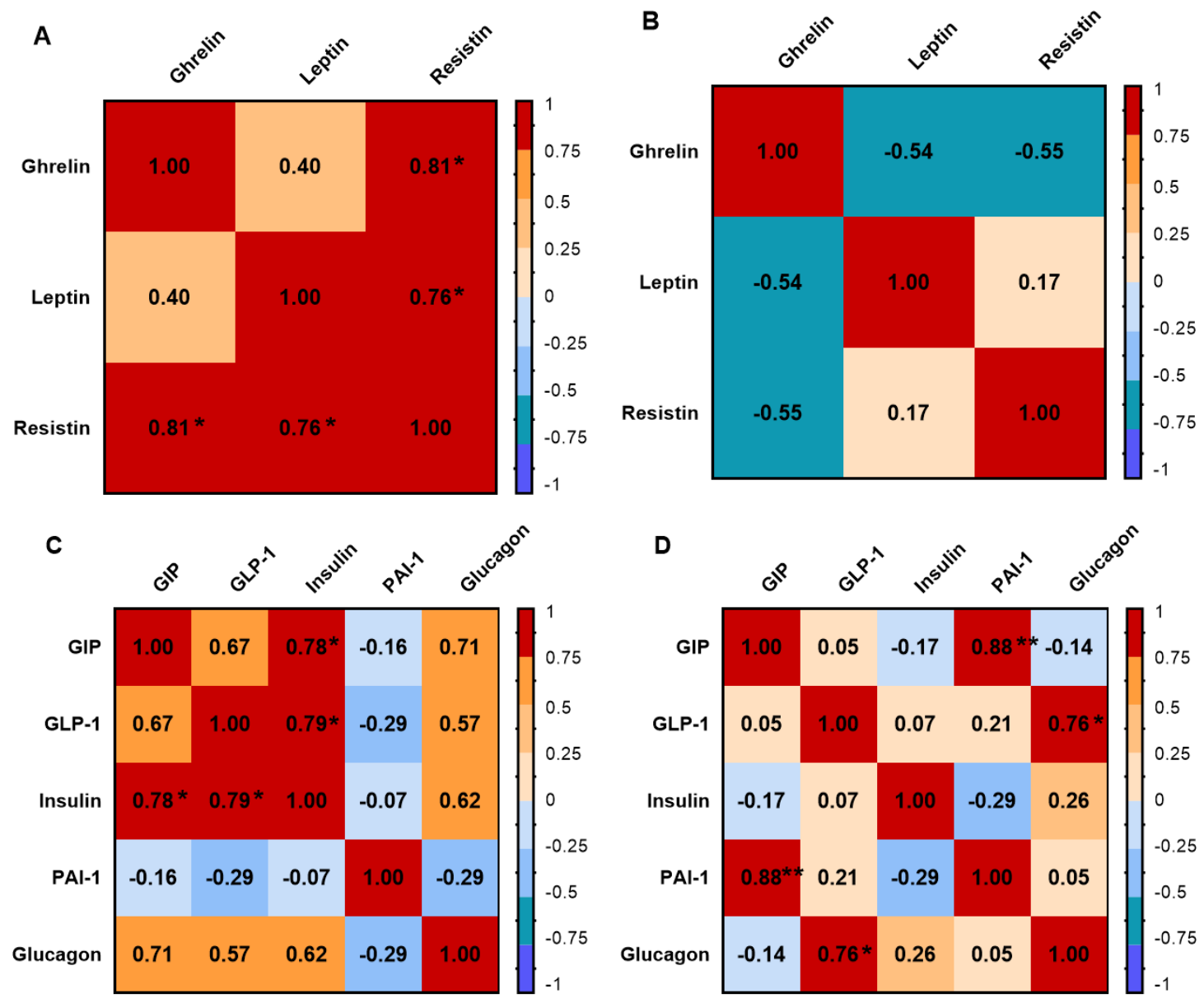

Figure 4. Spearman correlations for adipokines and metabolic biomarkers of insulin resistance. Red and blue squares refer to positive and negative correlations, respectively, with color intensity proportional to the correlation coefficient, as seen in the legend bar at the right. (A) Correlations of the adipokines ghrelin, resistin, and leptin in WT mice treated with leptin and VH. ${ }^{*} p<0.05$. (B) Correlation of the adipokines ghrelin, resistin, and leptin in TDP-43 ${ }^{\mathrm{A} 315 \mathrm{~T}}$ mice treated with leptin and VH. (C) Correlations for the metabolic biomarkers studied in WT mice treated with leptin and VH. ${ }^{*} p<0.05$. (D) Correlations for the metabolic biomarkers studied in TDP-43 ${ }^{\mathrm{A} 315 \mathrm{~T}}$ mice treated with leptin and VH. $* \mathrm{p}<0.05, * * \mathrm{p}<0.01$

\section{DISCUSSION}

The underlying pathogenesis of ALS is not fully understood and no cure has been discovered to either stop the neurodegeneration or to improve the lives of ALS patients. The risk of suffering this disease as well as its prognosis and progression, have been 
related to metabolic alterations. In fact, the majority of ALS patients suffer from malnutrition, which has been identified as a negative prognostic factor $[10,11]$, and is associated with low levels of leptin [12]. There is a direct association between leptin levels and survival in ALS patients [24]. However, the possible therapeutic effect of leptin treatment has not been explored. In this study, we sought to investigate the effects of leptin treatment in TDP-43 ${ }^{\mathrm{A} 315 \mathrm{~T}}$ mice, which develop neuropathology and behavioral deficits similar to human ALS.

Our results showed that transgenic animals treated with leptin had an earlier disease onset and, consequently, a longer disease duration. Nevertheless, the decline in body mass observed in this group was less marked, compared to VH-treated mice. Moreover, our study showed that leptin significantly improves motor coordination, suggesting a less aggressive ALS phenotype. Although other preclinical studies had shown a motor improvement after a leptin-deficiency in a SOD-1 mouse model of ALS [29], this contradiction suggests that the beneficial effects observed are not due only to leptin per se but to its interaction with the genetic background of the mouse model used. Furthermore, it is worth noting that SOD1-causing mutations represent only a minor subset of ALS patients while TDP-43 pathology is observed in the vast majority ( 80\%) of both sporadic and familial ALS cases $[9,30]$. Thus, leptin treatment could be beneficial for the majority of ALS patients.

Our results demonstrated that leptin treatment had a significant effect on the regulation of the expression of certain adipokines and metabolic proteins in TDP-43 ${ }^{\mathrm{A} 315 \mathrm{~T}}$ mice. The results showed an up-regulation of ghrelin in TDP-43 ${ }^{\mathrm{A} 315 \mathrm{~T}}$ after leptin treatment, which is an appetite-stimulating hormone. Low ghrelin plasmatic levels have been found in ALS patients [31], which may contribute to reduced total food intake, and consequently malnutrition observed in the majority of ALS patients [10, 11]. Ghrelin administration 
promotes food intake in humans [32]. Thus, it seems that the exogenous administration of leptin might induce hyperphagia, although through yet unknown mechanisms. This is consistent with the results showing that leptin treatment mitigates the sustained decline in body weight in TDP-43 ${ }^{\mathrm{A} 315 \mathrm{~T}}$ mice over time. Indeed, rapid weight loss in patients with ALS is associated with worse disease outcomes [25], therefore this slower body weight loss might contribute to a better disease progression.

Additionally, our results provide evidence of a lower expression of the adipokines resistin and leptin in TDP-43 ${ }^{\mathrm{A} 315 \mathrm{~T}}$ mice compared to controls WT animals. This is in contrast to other studies, which have shown no differences in ALS patients [31]. Moreover, there was an up-regulation of plasmatic leptin levels in WT animals treated with leptin, although it didn't reach significance, which was not observed in Lep-treated TDP-43 ${ }^{\text {A315T }}$ mice. Therefore, it seems that leptin treatment causes a decrease in endogenous leptin levels in transgenic ALS mice, however, a significant up-regulation of PAI-1 and GIP was observed in TDP-43 ${ }^{\mathrm{A} 315 \mathrm{~T}}$ mice after leptin treatment.

PAI-1 is a metabolic protein associated with an increase in adiposity and body mass index (BMI) [31, 33]. Other studies have shown that leptin upregulates the expression of PAI1 in vitro via activation of ERK1/2 [34], and the fact that endogenous leptin levels are low in these animals suggests that this effect is entirely due to the treatment with exogenous leptin. Moreover, since BMI is negatively correlated with ALS prognosis [9], this increase in PIA-1 might contribute to the neuroprotective role of leptin. Regarding GIP, low levels of this protein have been reported in ALS patients [31]. This protein stimulates insulin secretion in response to food intake [35] and it has been proposed as an obesity-promoting hormone [36]. Thus, once again, the up-regulation of GIP in TDP$43^{\mathrm{A} 315 \mathrm{~T}}$ mice in response to leptin treatment would help to reduce disturbances in energy metabolism and body weight loss associated with the progression of ALS. 
Finally, a positive correlation was found among ghrelin and leptin relative to resistin levels in the WT animals, which is related to insulin resistance [37]. Likewise, a positive correlation was found among the plasmatic levels of GIP and GLP-1 relative to insulin concentrations in the WT genotype, consistent with their role as incretins [38]. Nevertheless, these correlations are not found in the TDP-43 ${ }^{\mathrm{A} 315 \mathrm{~T}}$ genotype, indicating a possible development of insulin resistance in this ALS model. On the other hand, a positive correlation was found between levels of GIP and PAI-1 as well as between GLP1 and glucagon in TDP-43 $3^{\mathrm{A} 315 \mathrm{~T}}$ mice, in agreement with the metabolic impairments observed in ALS patients [39, 40].

In summary, although leptin treatment has been studied, and has shown beneficial effects in relationship with a wide range of pathologies such as lipodystrophy, obesity, type 2 diabetes [41], depression [42], or Alzheimer's disease [23], this is, to our knowledge, the first preliminary experimental evidence for the beneficial effect of leptin in treating ALS. However, future studies will be necessary to further prove this effect and to determine the specific pathway through which leptin performs this therapeutic action.

\section{MATERIAL AND METHODS}

\subsection{Animals}

Transgenic TAR DNA-binding protein 43 (TDP43 ${ }^{\mathrm{A} 315 \mathrm{~T}}$ ) mice [43] and age and gendermatched wild-type littermates (WT, controls) (Strain No. 010700, Bar Harbor, ME, USA) were used in this study. To generated this mouse model of ALS, the mouse prion promoter (Prt) and a cDNA encoding human TARDBP with an A315T mutation (hTDP-43 ${ }^{\mathrm{A} 315 \mathrm{~T}}$ ) associated with fALS and containing an $\mathrm{N}$ terminal FLAG-tag were used [43]. These mice accumulate cytoplasmic TDP-43 in the brain and spinal cord, creating TDP-43 aggregates and developing neuropathology and behavioural deficits similar to ALS [44], 
they do not, however, develop neuronal loss or paralysis [43]. TDP43 ${ }^{\mathrm{A} 315 \mathrm{~T}}$ mice were used as hemizygotes, therefore the progeny was genotyped at 21 days of age by detecting hTDP-43 transgene using standard tail genomic DNA PCR analysis according to the distributor's protocol.

Only male mice were used, to avoid the reported gender-related differences in mean survival time of TDP-43 ${ }^{\mathrm{A} 315 \mathrm{~T}}$ mice $[43,45]$. Animals were group-housed under standard housing conditions with a $12 \mathrm{~h}$ light-dark cycle, with food and water available ad libitum, and their general health was regularly checked. Additionally, TDP-43 ${ }^{\mathrm{A} 315 \mathrm{~T}}$ mice were fed jellified food in pellet form (D5K52 food. Rettenmaier Ibérica, Spain) to mitigate the intestinal dysmotility phenotype and sudden death [46].

\subsection{Monitoring and behavioral assessments}

To monitor disease progression, all mice were weighed and assessed three times per week until the disease onset-stage, defined as the last day of individual peak body weight before a gradual loss occurs. After that, mice were weight daily until the disease end-stage, defined as three consecutive days with a weight below $80 \%$ of the initial weight.

To evaluate motor performance, the accelerated rota-rod protocol was used on all mice once a week $[47,48]$, starting at 7 weeks of age until the disease end-stage. Animals were previously trained for three consecutive days and three times a day to promote the learning of the task. Briefly, mice were placed on a rota-rod apparatus (Model 7650, Ugo Basile) at a speed of $4 \mathrm{rpm}$ with acceleration up to $40 \mathrm{rpm}$ over $300 \mathrm{~s}$. Each mouse performed three tests with a minimal interval of $20 \mathrm{mins}$, and the average of the longest two performances was taken as the final result for analysis. Lower scores on the rota-rod are indicative of impaired locomotor function.

\subsection{Drug Administration}


Male TDP-43 ${ }^{\mathrm{A} 315 \mathrm{~T}}$ mice and WT littermates were divided into two subgroups $(\mathrm{n}=3-5 \mathrm{Tg}$ mice/ subgroup and $n=4$ WT mice/ subgroup) according to the treatments, resulting in four groups in total. At 6 weeks of age, animals were treated intranasally (IN) with leptin or vehicle (VH) daily for 7 days, as previously described [17]. No difference in body weight gain between groups was observed during the treatment period (Table 1).

\begin{tabular}{|c|c|c|c|c|}
\hline & Control VH $(n=4)$ & Control Lep $(n=4)$ & TDP-43 ${ }^{\mathrm{A315T}}$ VH $(n=5)$ & TDP $-43^{\mathrm{A315T}}$ Lep $(n=3)$ \\
\hline 1d & $20,09 \pm 0.20$ & $21,1 \pm 0.65$ & $18,93 \pm 0.49$ & $20,73 \pm 0.48$ \\
\hline 2d & $20,63 \pm 0.35$ & $20,73 \pm 0.56$ & $18,8 \pm 0.78$ & $20,86 \pm 0.18$ \\
\hline 3d & $21,63 \pm 0.32$ & $20,8 \pm 0.47$ & $18,87 \pm 0.83$ & $20,96 \pm 0.32$ \\
\hline 4d & $21,9 \pm 0.43$ & $20,8 \pm 0.44$ & $19,03 \pm 0.77$ & $20,93 \pm 0.35$ \\
\hline 5d & $21,87 \pm 0.51$ & $20,85 \pm 0.69$ & $19,4 \pm 0.62$ & $20,5 \pm 0.53$ \\
\hline 6d & $22,13 \pm 0.47$ & $21,3 \pm 0.54$ & $19,5 \pm 0.69$ & $21 \pm 0.56$ \\
\hline $7 d$ & $21,65 \pm 0.26$ & $21,4 \pm 0.61$ & $19,22 \pm 0.63$ & $21,23 \pm 0.54$ \\
\hline
\end{tabular}

Table 1. No difference in body weight gain was observed during leptin treatment. Body weight was monitored daily in WT and TDP-43 ${ }^{\mathrm{A} 315 \mathrm{~T}}$ mice during the 7-days treatment period and no difference in body weight gain between treatments and groups was observed. Values are expressed as mean \pm SEM.

Then, a leptin solution was prepared in $0.125 \%(2.3 \mathrm{mM})$ of N-tetradecyl-b-D maltoside (TDM) (Sigma-Aldrich) reconstituted in PBS (pH 7.2) at a concentration of $1 \mathrm{mg} / \mathrm{mL}$ and IN treatments were given under light anesthesia ( $3 \%$ isoflurane), using a $30 \mu \mathrm{L}$ pipette bearing a $20 \mu \mathrm{L}$ long gel loading tip to introduce $20 \mu \mathrm{L}$ of $0.03 \mathrm{mg} / \mathrm{kg}$ leptin or VH into the nasal cavity via one of the external nares. Nares were alternated each day during the week treatment period, starting with the left side.

The IN-administration method was selected as it has rapid substance absorption with minimal discomfort, and it enables the administration of smaller drug volumes than other 
non-invasive administration routes since it avoids the hepatic first-pass effect observed in oral delivery [49-51].

\subsection{Measurement of metabolic markers}

At disease end-stage ( 95-100 \pm 2 days), mice were terminally anesthetized with sodium pentobarbitone $(140 \mathrm{mg} / \mathrm{kg})$. Blood was collected by cardiac puncture and immediately centrifuged at $3380 \mathrm{~g}$ for $10 \mathrm{~min}$ at room temperature to obtain plasma, which was then frozen on dry ice and stored at $-80{ }^{\circ} \mathrm{C}$ for later analysis [52].

Plasma samples were used to measure adipokines (ghrelin, resistin, and leptin) and metabolic biomarkers of insulin resistance (GIP, GLP-1, Glucagon, Insulin, and PAI-1) using the Bio-PlexPro mouse Diabetes group (Bio-Rad, Ref. 171F7001M), in a Luminex® 200тм technology as previously described [53]. Samples were analyzed using duplicate analyses following the manufacturer's instructions. The final concentration value of each metabolic marker was the result of the mean from the two duplicated measures.

\subsection{Statistical analysis}

Data is expressed as means \pm standard error of the mean (SEM). A two-way ANOVA was used to assess differences between means over time in body weight and rota-rod performance, followed by a Tukey post-hoc analysis. A one-way ANOVA, followed by a Tukey post-hoc analysis, was used to assess differences between means in the plasmatic levels of metabolic biomarkers. A student t-test was used only when two groups were compared for disease onset and duration, assuming equal variances. A spearman correlation was performed for both the adipokines and metabolic biomarkers. A value of $p<0.05$ (CI 95\%) was considered statistically significant. All statistical analyses were performed using GraphPad Prism software (version 8.0.1). 
Funding: This work was supported by the funding from the Consejería de Educación, Cultura y Deportes, Fondo Europeo de Desarrollo Regional (FEDER), Junta de Comunidades de Castilla-La Mancha (SBPLY/17/180501/000303).

Acknowledgments: The authors would like to gratefully acknowledge the Animal Facility and Experimental Surgery Unit of the UDI-HNP for their excellent technical support.

Author contribution: Conceptualization, CM.F.-M.; Methodology, A.F.-D., P.F., CM.F.-M.; Formal Analysis, A.C., P.F., CM.F.-M.; Investigation, A.F.-D., CM.F.-M.; Writing, A.C.; Writing - Review \& Editing, A.C., CM.F.-M.; Supervision, CM.F.-M.; Project Administration, CM.F.-M.; Funding Acquisition, CM.F.-M.; All authors have read and agreed to the published version of the manuscript.

Institutional Review Board Statement: All experimental procedures were approved by the Animal Ethics Committee of the National Hospital for Paraplegics (HNP) (Approval No. 26/OH 2018), in accordance with the Spanish Guidelines for the Care and Use of Animals for Scientific Purposes.

Informed Consent Statement: Not applicable.

Conflicts of Interest: The authors declare no conflict of interest. 


\section{REFERENCES}

1. Tapia, R. Cellular and molecular mechanisms of motor neuron death in amyotrophic lateral sclerosis: a perspective. Front Cell Neurosci 2014, 8, 241. Doi: 10.3389/fncel.2014.00241.

2. Longinetti, E.; Fang, F. Epidemiology of amyotrophic lateral sclerosis: An update of recent literature. Curr Opin Neurol 2019, 32(5), 771-776. Doi: 10.1097/WCO.0000000000000730.

3. Jaiswal, M.K. Riluzole and edaravone: A tale of two amyotrophic lateral sclerosis drugs. Med Res Rev 2019, 39(2), 733-748. Doi: 10.1002/med.21528.

4. Nowicka, N.; Juranek, J.; Juranek, J.K.; Wojtkeiwicz, J. Risk Factors and Emerging Therapies in Amyotrophic Lateral Sclerosis. Int J Mol Sci 2019, 20(11), 2616. Doi: 10.3390/ijms20112616.

5. Vandoorne, T.; De Bock, K.; Van Den Bosch, L. Energy metabolism in ALS: an underappreciated opportunity? Acta Neuropathol 2018, 135(4), 489-509. Doi: 10.1007/s00401-018-1835-x.

6. Steyn, F.J.; Ioannides, Z.A.; Van Eijk, R.P.A.; Heggie, S.; Thorpe, K.A.; Ceslis, A.; et al. Hypermetabolism in ALS is associated with greater functional decline and shorter survival. J Neurol Neurosurg Psychiatry 2018, 89(10), 1016-1023. Doi: 10.1136/jnnp2017-317887.

7. Diekmann, K.; Kuzma-Kozakiewicz, M.; Piotrkiewicz, M.; Gromicho, M.; Grosskreutz, J.; Andersen, P.M.; et al. Impact of comorbidities and co-medication on disease onset and progression in a large German ALS patient group. J Neurol 2020, 267(7), 2130-2141. Doi: 10.1007/s00415-020-09799-z.

8. Paccosi, S.; Cresci, B.; Pala, L.; Rotella, C.M.; Parenti, A. Obesity Therapy: How and Why? Curr Med Chem 2020, 27(2), 174-186. Doi: 10.2174/0929867326666190124121725.

9. Pape, J.A.; Grose, J.H. The effects of diet and sex in amyotrophic lateral sclerosis. Rev Neurol (Paris) 2020, 176(5), 301-315. Doi: 10.1016/j.neurol.2019.09.008.

10. Ludolph, A.C.; Dorst, J.; Dreyhaupt, J.; Weishaupt, J.H.; Kassubek, J.; Weiland, U.; et al. Effect of High-Caloric Nutrition on Survival in Amyotrophic Lateral Sclerosis. Ann Neurol 2020, 87(2), 206-216. Doi: 10.1002/ana.25661.

11. Lopez-Gomez, J.J.; Ballesteros-Pomar, M.D.; Torres-Torres, B.; Pintor de la Maza, B.; Penacho-Lázaro, M.A.; Palacio-Mures, J.M.; et al. Malnutrition at diagnosis in amyotrophic lateral sclerosis (ALS) and its influence on survival: Using glim criteria. Clin Nutr 2021, 40(1), 237-244. Doi: 10.1016/j.clnu.2020.05.014.

12. Cohen, S.; Danzaki, K.; MacIver, N.J. Nutritional effects on T-cell immunometabolism. Eur J Immunol 2017, 47(2), 225-235. Doi: 10.1002/eji.201646423. 
13. Zhang, Y.; Proenca, R.; Maffei, M.; Barone, M.; Leopold, L.; Friedman, J.M. Positional cloning of the mouse obese gene and its human homologue. Nature 1994, 372(6505), 425-432. Doi: 10.1038/372425a0.

14. Stephens, T.W.; Basinski, M.; Bristow, P.K.; Bue-Valleskey, J.M.; Burgett, S.G.; Craft, L.; et al. The role of neuropeptide $\mathrm{Y}$ in the antiobesity action of the obese gene product. Nature 1995, 377(6549), 530-532. Doi: 10.1038/377530a0.

15. King, A.; Brain, A.; Hanson, K.; Dittmann, J.; Vickers, J.; Fernandez-Martos, C. Disruption of leptin signalling in a mouse model of Alzheimer's disease. Metab Brain Dis 2018, 33(4), 1097-1110. Doi: 10.1007/s11011-018-0203-9.

16. Perez-Gonzalez, R.; Alvira-Botero, M.X.; Robayo, O.; Antequera, D.; Garzon, M.; Martin-Moreno, A.M.; et al. Leptin gene therapy attenuates neuronal damages evoked by amyloid-beta and rescues memory deficits in APP/PS1 mice. Gene Ther 2014, 21(3), 298-308. Doi: 10.1038/gt.2013.85.

17. Fernandez-Martos, C.M.; Atkinson, R.A.K.; Chuah, M.I.; King, A.E.; Vickers, J.C. Combination treatment with leptin and pioglitazone in a mouse model of Alzheimer's disease. Alzheimer's Dement TRCI 2017, 3(1), 92-106. Doi: 10.1016/j.trci.2016.11.002.

18. Weng, Z.; Signore, A.P.; Gao, Y.; Wang, S.; Zhang, F.; Hastings, T.; et al. Leptin protects against 6-hydroxydopamine-induced dopaminergic cell death via mitogenactivated protein kinase signaling. J Biol Chem 2007, 282(47), 34479-91. Doi: 10.1074/jbc.M705426200.

19. Zhang, F.; Wang, S.; Signore, A.P.; Chen, J. Neuroprotective effects of leptin against ischemic injury induced by oxygen-glucose deprivation and transient cerebral ischemia. Stroke 2007, 38(8), 2329-36. Doi: 10.1161/STROKEAHA.107.482786.

20. Fernandez-Martos, C.M.; Gonzalez, P.; Rodriguez, F.J. Acute leptin treatment enhances functional recovery after spinal cord injury. PLoS One 2012, 7(4), e35594. Doi: 10.1371/journal.pone.0035594.

21. Farr, S.A.; Banks, W.A.; Morley, J.E. Effects of leptin on memory processing. Peptides 2006, 27(6), 1420-5. Doi: 10.1016/j.peptides.2005.10.006.

22. Moult, P.R.; Milojkovic, B.; Harvey, J. Leptin reverses long-term potentiation at hippocampal CA1 synapses. J Neurochem 2009, 108(3), 685-96. Doi: 10.1111/j.14714159.2008.05810.x.

23. Liu, Y.; Hanson, K.A.; McCormack, G.; Atkinson, R.A.K.; Dittmann, J.; Vickers, J.C.; et al. Enhanced Anti-Amyloid Effect of Combined Leptin and Pioglitazone in APP/PS1 Transgenic Mice. Curr Alzheimer Res 2020, 17(14), 1294-1301. Doi: 10.2174/1567205018666210218163857.

24. Nagel, G.; Peter, R.S.; Rosenbohm, A.; Koenig, W.; Dupuis, L.; Rothenbacher, D.; et al. Adipokines, C-reactive protein, and Amyotrophic Lateral Sclerosis - results from a population-based ALS registry in Germany. Sci Rep 2017, 7(1), 4374. Doi: 10.1038/s41598-017-04706-5. 
25. Ahmed, R.M.; Phan, K.; Highton-Williamson, E.; Strikwerda-Brown, C.; Caga, J.; Ramsey, E.; et al. Eating peptides: biomarkers of neurodegeneration in amyotrophic lateral sclerosis and frontotemporal dementia. Ann Clin Transl Neurol 2019, 6(3), 486495. Doi: 10.1002/acn3.721.

26. Dupuis, L.; Oudart, H.; Rene, F.; Gonzalez de Aguilar, J.L.; Loeffler, J.P. Evidence for defective energy homeostasis in amyotrophic lateral sclerosis: benefit of a highenergy diet in a transgenic mouse model. Proc Natl Acad Sci USA 2004, 101(30), 1115964. Doi: 10.1073/pnas.0402026101.

27. Wills, A.M.; Hubbard, J.; Macklin, E.A.; Glass, J.; Tandan, R.; Simpson, E.P.; et al. Hypercaloric enteral nutrition in patients with amyotrophic lateral sclerosis: a randomised, double-blind, placebo-controlled phase 2 trial. Lancet 2014, 383(9934), 2065-72. Doi: 10.1016/S0140-6736(14)60222-1.

28. Fasshauer, M.; Blüher, M. Adipokines in health and disease. Trends Pharmacol Sci 2015, 36(7), 461-70. Doi: 10.1016/j.tips.2015.04.014.

29. Lim, M.A.; Bence, K.K.; Sandesara, I.; Andreux, P.; Auwerx, J.; Ishibashi, J.; et al. Genetically altering organismal metabolism by leptin-deficiency benefits a mouse model of amyotrophic lateral sclerosis. Hum Mol Genet 2014, 23(18), 4995-5008. Doi: $10.1093 / \mathrm{hmg} / \mathrm{ddu} 214$.

30. Chen-Plotkin, A.S.; Lee, V.M.; Trojanowski, J.Q. TAR DNA-binding protein 43 in neurodegenerative disease. Nat Rev Neurol 2010, 6(4), 211-20. Doi: 10.1038/nrneurol.2010.18.

31. Ngo, S.T.; Steyn, F.J.; Huang, L.; Mantovani, S.; Pfluger, C.M.M.; Woodruff, T.M.; et al. Altered expression of metabolic proteins and adipokines in patients with amyotrophic lateral sclerosis. $J$ Neurol Sci 2015, 357(1-2), 22-7. Doi: 10.1016/j.jns.2015.06.053.

32. Wren, A.M.; Seal, L.J.; Cohen, M.A.; Brynes, A.E.; Frost, G.S.; Murphy, K.G.; et al. Ghrelin enhances appetite and increases food intake in humans. J Clin Endocrinol Metab 2001, 86(12), 5992. Doi: 10.1210/jcem.86.12.8111.

33. Kahn, S.E.; Zinman, B.; Haffner, S.M.; O'Neill, M.C.; Kravitz, B.G.; Yu, D.; et al. Obesity is a major determinant of the association of c-reactive protein levels and metabolic syndrome in type 2 diabetes. Diabetes 2006, 55(8), 2357-2364. Doi: $10.2337 / \mathrm{db} 06-0116$.

34. Singh, P.; Peterson, T.E.; Barber, K.R.; Kuniyoshi, F.S.; Jensen, A.; Hoffmann, M.; et al. Leptin upregulates the expression of plasminogen activator inhibitor-1 in human vascular endothelial cells. Biochem Biophys Res Commun 2010, 392(1), 47-52. Doi: 10.1016/j.bbrc.2009.12.158.

35. Elliott, R.M.; Morgan, L.M.; Tredger, J.A.; Deacon, S.; Wright, J.; Marks, V. Glucagon-like peptide-1 (7-36) amide and glucose-dependent insulinotropic polypeptide secretion in response to nutrient ingestion in man: acute post-prandial and 24-h secretion patterns. J Endocrinol 1993, 138(1), 159-166. Doi: 10.1677/joe.0.1380159. 
36. Holst, J.J.; Rosenkilde, M.M. Recent advances of GIP and future horizons. Peptides 2020, 125, 170230. Doi: 10.1016/j.peptides.2019.170230.

37. Meier, U.; Gressner, A.M. Endocrine regulation of energy metabolism: review of pathobiochemical and clinical chemical aspects of leptin, ghrelin, adiponectin, and resistin. Clin Chem 2004, 50(9), 1511-25. Doi: 10.1373/clinchem.2004.032482.

38. Nauck, M.A.; Meier, J.J. Incretin hormones: Their role in health and disease. Diabetes Obes Metab 2018, 20(1), 5-21. Doi: 10.1111/dom.13129.

39. Dodge, J.C.; Treleaven, C.M.; Fidler, J.A.; Tamsett, T.J.; Bao, C.; Searles, M.; et al. Metabolic signatures of amyotrophic lateral sclerosis reveal insights into disease pathogenesis. Proc Natl Acad Sci USA 2013, 110(26), 10812-7. Doi: 10.1073/pnas.1308421110.

40. Tefera, T.W.; Steyn, F.J.; Ngo, S.T.; Borges, K. CNS glucose metabolism in Amyotrophic Lateral Sclerosis: a therapeutic target? Cell Biosci 2021, 11(1), 14. Doi: 10.1186/s13578-020-00511-2.

41. Paz-Filho, G.; Mastronardi, C.A.; Licinio, J. Leptin treatment: facts and expectations. Metabolism 2015, 64(1), 146-56. Doi: 10.1016/j.metabol.2014.07.014.

42. Ge, T.; Fan, J.; Yang, W.; Cui, R.; Li, B. Leptin in depression: a potential therapeutic target. Cell Death Dis 2018, 9(11), 1096. Doi: 10.1038/s41419-018-1129-1.

43. Wegorzewska, I.; Bell, S.; Cairns, N.J.; Miller, T.M.; Baloh, R.H. TDP-43 mutant transgenic mice develop features of ALS and frontotemporal lobar degeneration. Proc Natl Acad Sci USA 2009, 106(44), 18809-18814. Doi: 10.1073/pnas.0908767106.

44. Ke, Y.D.; van Hummel, A.; Stevens, C.H.; Gladbach, A.; Ippati, S.; Bi, M.; et al. Short-term suppression of a315t mutant human TDP-43 expression improves functional deficits in a novel inducible transgenic mouse model of FTLD-TDP and ALS. Acta Neuropathol 2015, 130(5), 661-678. Doi: 10.1007/s00401-015-1486-0.

45. Hatzipetros, T.; Bogdanik, L.P.; Tassinari, V.R.; Kidd, J.D.; Moreno, A.J.; Davis, C.; et al. C57bl/6j congenic prp-TDP43a315t mice develop progressive neurodegeneration in the myenteric plexus of the colon without exhibiting key features of ALS. Brain Res 2014, 1584, 59-72. Doi: 10.1016/j.brainres.2013.10.013.

46. Herdewyn, S.; Cirillo, C.; Van Den Bosch, L.; Robberecht, W.; Vanden Berghe, P.; Van Damme, P. Prevention of intestinal obstruction reveals progressive neurodegeneration in mutant TDP-43 (a315t) mice. Mol Neurodegener 2014, 9, 24. Doi: 10.1186/1750-1326-9-24.

47. Mandillo, S.; Tucci, V.; Holter, S.M.; Meziane, H.; Banchaabouchi, M.A.; Kallnik, M.; et al. Reliability, robustness, and reproducibility in mouse behavioral phenotyping: A cross-laboratory study. Physiol Genomics 2008, 34(3), 243-255. Doi: 10.1152/physiolgenomics.90207.2008.

48. Dang, T.N.; Lim, N.K.; Grubman, A.; Li, Q.X.; Volitakis, I.; White, A.R.; et al. Increased metal content in the tdp-43(a315t) transgenic mouse model of frontotemporal 
lobar degeneration and amyotrophic lateral sclerosis. Front Aging Neurosci 2014, 6, 15. Doi: 10.3389/fnagi.2014.00015.

49. Turner, C.L.; Eggleston, G.W.; Lunos, S.; Johnson, N.; Wiedmann, T.S.; Bowles, W.R. Sniffing out endodontic pain: use of an intranasal analgesic in a randomized clinical trial. J Endod 2011, 37(4), 439-44. Doi: 10.1016/j.joen.2010.12.010.

50. Rosseels, V.; Naze, F.; De Craeye, S.; Francart, A.; Kalai, M.; Van Gucht, S. A noninvasive intranasal inoculation technique using isoflurane anesthesia to infect the brain of mice with rabies virus. $J$ Virol Methods 2011, 173(1), 127-36. Doi: 10.1016/j.jviromet.2011.01.019.

51. Wu, S.; Li, K.; Yan, Y.; Gran, B.; Han, Y.; Zhou, F.; et al. Intranasal Delivery of Neural Stem Cells: A CNS-specific, Non-invasive Cell-based Therapy for Experimental Autoimmune Encephalomyelitis. J Clin Cell Immunol 2013, 4(3). Doi: 10.4172/21559899.1000142.

52. Rodriguez, A.; Ferrer-Donato, A.; Cabrera-Pinto, M.; Seseña, S.; Fernández, P.; Aranda, A.; et al. Effect of ozone exposure on Amyotrophic Lateral Sclerosis (ALS) pathology using a mice model of TDP-43 proteinopathy. bioRxiv 2021, 430915 Doi: 10.1101/2021.02.12.430915.

53. Ortega-Moreno, L.; Sanz-Garcia, A.; Fernandez de la Fuente, M.J.; Arroyo Solera, R.; Fernandez-Tome, S.; Marin, A.C.; et al. Serum adipokines as non-invasive biomarkers in Crohn's disease. Sci Rep 2020, 10(1), 18027. Doi: 10.1038/s41598-020-74999-6. 